\title{
Wahlpflicht und Demokratie
}

\section{Zur Geschichte eines Problems: die Wahlpflicht als ein Prüfstein der Demokratietheorie}

Wenn man sich vornimmt, einen Aufsatz zu einem Thema zu schreiben, über das schon sehr vieles gesagt worden ist, sollte man mit Neuigkeiten beginnen. Das Thema, das wir in diesem Aufsatz behandeln, ist die Frage nach der Vereinbarkeit einer Wahlpflicht mit dem Wesen der Demokratie. Die Neuigkeit, die wir dabei in das Zentrum der Überlegungen stellen, ist die These, dass es bei diesem Thema um ein verfassungstheoretisches Grundproblem geht: um das Grundproblem nämlich, wie die abstrakte Idee einer bestimmten verfassungsrechtlichen Ordnung an die konkret existierende Menschengemeinschaft angeknüpft werden kann.

Um Schritt für Schritt zu diesem Punkt zu gelangen und hieraus juristische Schlüsse zu ziehen, müssen wir zunächst die Hintergründe der Wahlpflichtfrage beleuchten. Anzutreffen war die Idee einer Wahlpflicht bereits im vorletzten Jahrhundert. ${ }^{1}$ Bis heute kennt man eine solche Pflicht, deren Verletzung das Recht sanktioniert, beispielsweise in Belgien, Luxemburg und in Australien. ${ }^{2}$ Auch Österreich ${ }^{3}$ und die Schweiz ${ }^{4}$ verfügen in Sachen Wahlpflicht über eine reiche Erfahrung, da eine solche Pflicht in verschiedenen österreichischen Ländern und schweizerischen Kantonen über Jahrzehnte hinweg festgelegt war und praktiziert worden ist. In Deutschland wurde die Einführung einer Wahlpflicht schon des öfteren diskutiert, ${ }^{5}$ ohne dass ein solcher Vorschlag (abgesehen

1 Die Geschichte der Wahlpflichtidee im 19. Jahrhundert wurde von den damaligen Autoren detailliert dokumentiert; vgl. Heinrich Geffcken, ZfP 2 (1909), 159, 160 ff.; William A. Robson, Political Science Quarterly 38 (1923), 569, 570 ff.; Heinrich Triepel, Wahlrecht und Wahlpflicht, S. 12 ff. und Alexander Vutkovich, Wahlpflicht, S. 9 ff.

2 Einen umfassenden Überblick dazu verschaffen Felix Ermacora, Allgemeine Staatslehre, Erster Teilband, S. 465 f.; Maria Gratschew, in: International Institute for Democracy and Electoral Assistance (Hrsg.), Voter Turnout, S. 105; dies., in: International Institute for Democracy and Electoral Assistance (Hrsg.), Voter Turnout in Western Europe, S. 25; Richard L. Hasen, University of Pennsylvania Law Review 144 (1996), 2135, 2169 ff. und Sean Matsler, Southern California Law Review 76 (2003), 953, 962 ff.

3 Zur Geschichte der Wahlpflicht in Österreich Walter Berka, VVDStRL 55 (1996), 48, 52 und Manfred Nowak, Politische Grundrechte, S. 389 ff.; zum 19. Jahrhundert bereits Heinrich Geffcken, ZfP 2 (1909), 159, 168 f. und Alexander Vutkovich, Wahlpflicht, S. 49 ff. Zur Wirkung der Wahlpflicht in Österreich aus politikwissenschaftlicher Sicht Wolfgang Hirczy, Electoral Studies 13 (1994), 64.

4 Zur Geschichte der Wahlpflicht in der Schweiz Alois Riklin, in: Festschrift für Kurt Eichenberger, S. 41, 52; zum 19. Jahrhundert wiederum Heinrich Geffcken, ZfP 2 (1909), 159, 163 f; Heinrich Triepel, Wahlrecht und Wahlpflicht, S. 15 ff. und Alexander Vutkovich, Wahlpflicht, S. $49 \mathrm{ff}$.

5 Vgl. dazu Klaus Stern, Das Staatsrecht der Bundesrepublik Deutschland, Bd. I, 2. Auflage, S. 322; ausführlich zur Geschichte des Wahlpflichtgedankens in Deutschland Ernst Rudolf Huber, Deutsche Verfassungsgeschichte, Bd. III, 3. Auflage, S. 863 ff. Die Entwicklung im 19. Jahrhundert dokumentiert Alexander Vutkovich, Wahlpflicht, S. $31 \mathrm{ff}$. 
von vereinzelten Experimenten in den Kleinstaaten des 19. und des frühen 20. Jahrhunderts) umgesetzt worden wäre. ${ }^{6}$ Den einzigen Hinweis auf eine Wahlpflicht bildet im geltenden Recht Art. 26 Abs. 3 der baden-württembergischen Landesverfassung, der die Ausübung des Wahl- und Stimmrechts eine „Bürgerpflicht" ${ }^{\text {"7 }}$ nennt, freilich ohne diese „Pflicht" in irgendeiner Form zu sanktionieren. ${ }^{8}$ Als Gegenstand von verfassungsrechtlichen Reflexionen ist das Thema Wahlpflicht demnach alt. ${ }^{9}$ Dennoch lohnt es sich gerade heute, aus juristischer Sicht darüber zu sprechen, und hierfür gibt es drei Gründe.

Der erste dieser Gründe ist rechtspraktischer Art. Trotz seiner jahrhundertelangen und wechselvollen Geschichte ist der Gegenstand Wahlpflicht politisch noch nicht überholt: gefordert wird die Einführung einer entsprechenden Pflicht praktisch nach jeder Wahl, bei der die Wahlbeteiligung niedrig ausfällt. Zuletzt angeregt wurde dies nach den Europawahlen 2009, ${ }^{10}$ bei welchen die Wahlbeteiligung im bundesweiten Durchschnitt nur bei knapp über 43 Prozent, im Bundesland mit der niedrigsten Wahlbeteiligung sogar nur bei 30 Prozent lag. ${ }^{11}$ Unterstützt wird die Forderung nach der Einführung einer Wahlpflicht auch von wissenschaftlicher Seite. ${ }^{12}$

Der zweite Grund, der es lohnenswert macht, sich als Jurist mit dem Wahlpflichtproblem zu befassen, ist ein erkenntnisstrategischer Grund - er folgt aus dem Wesen der Fragen, um die es bei diesem Gegenstand geht. Um zu analysieren, ob die Einführung einer Wahlpflicht mit dem Grundsatz der Freiheit der Wahl zu vereinbaren ist, bedarf

6 Die Behauptung, dass in der DDR (ebenso wie in den anderen Staaten des ehemaligen Ostblocks) eine faktische Wahlpflicht bestand (so statt vieler Otto Luchterhandt, Grundpflichten als Verfassungsproblem in Deutschland, S. 498 und Thorsten Ingo Schmidt, Grundpflichten, S. 218 ff.), ist nicht haltbar. Das Bestehen einer Wahlpflicht setzt voraus, dass Wahlen stattfinden, was in keinem dieser Staaten der Fall war.

7 Dass sich eine solche „Bürgerpflicht“, an der Wahl teilzunehmen, rational begründen lässt, bestreiten aus Sicht der Politikwissenschaften Loren E. Lomasky/Geoffrey Brennan, Social Philosophy \& Policy 17 (2000), 62; vgl. auch Wyndraeth H. Morris Jones, Political Studies 2 (1954), 25.

8 Zu dieser Vorschrift Klaus Braun, Kommentar zur Verfassung des Landes Baden-Württemberg, Rn. 9 zu Art. 26; Detlef Merten, VVDStRL 55 (1996), 7, 24 und Thorsten Ingo Schmidt, Grundpflichten, S. 219.

9 Siehe dazu aus der rechtswissenschaftlichen Literatur: Walter Frenz, ZRP 1994, 91; Richard L. Hasen, University of Pennsylvania Law Review 144 (1996), 2135, 2169 ff.; Heather Lar$d y$, Oxford Journal of Legal Studies 24 (2004), 303; Sean Matsler, Southern California Law Review 76 (2003), 953 und Detlef Merten, in: Festschrift für Johannes Broermann, S. 301; aus der politikwissenschaftlichen Literatur u.a. Lisa Hill, Political Studies 50 (2002), 80; dies., Journal of Sociology 36 (2000), 30 und Arend Lijphart, American Political Science Review 91 (1997), 1.

10 Vgl. hierzu die Meldung des Nachrichtenportals Spiegel Online vom 9.6.2009: http:// www.spiegel.de/politik/deutschland/0,1518,629335,00.html.

11 Die Zahlenangaben wurden der Statistik entnommen, die vom Bundeswahlleiter im Internet publiziert worden ist; vgl. http://www.bundeswahlleiter.de/de/europawahlen/ EU_BUND_09/ergebnisse/index.html.

12 Siehe dazu die Äußerungen des Grazer Soziologen Max Haller, welche die Online-Ausgabe des „Standard“ zitiert: http://derstandard.at/1237228645781?_seite=68csap=2, sowie das Interview des Nachrichtenportals Welt Online mit dem Wahlforscher Armin Schäfer vom 14.5.2010: http://www.welt.de/politik/nrw-wahl/article7627263/Forscher-fordert-neue-Diskussion-ueber-Wahlpflicht.html. 
es der Klarheit über die Rechtsnatur der Befugnis zu wählen, die eingeordnet werden muss in den großen Fragenkreis des Zusammenhangs von Freiheit und Demokratie. ${ }^{13}$ Gerade dieses Verhältnis jedoch ist es, das heutzutage thematisiert werden muss, wenn es darum geht, die Zukunft der demokratischen Herrschaft in einer Ordnung zu untersuchen, in welcher der klassische Nationalstaat seine Bedeutung möglicherweise verliert. Das Demokratiekonzept, das wir im folgenden darlegen werden, ist insofern auch als ein Beitrag zur Diskussion über die Zukunft der demokratischen Herrschaft zu sehen.

Hierbei zeigt es sich nun, dass die Herangehensweise an diese Fragen innerhalb der zurückliegenden Jahre eine andere wurde, so dass auch die verfassungsrechtliche $\mathrm{Zu}$ lässigkeit einer Wahlpflicht heutzutage von zahlreichen Autoren anders eingeschätzt wird, als dies in den vorangegangenen Jahrzehnten mehrheitlich getan worden ist. Dies genau ist der dritte Grund, der es erfordert, das Thema Wahlpflicht zu untersuchen. Seiner Natur nach ist er rechtswissenschaftlicher Art: zu lösen bleibt ein Verfassungsproblem. Zur Vereinbarkeit einer Wahlpflicht mit der Freiheit der Wahl (und demnach: mit dem Wesen der Demokratie) hatten sich bereits in der Vergangenheit zahlreiche prominente Vertreter der deutschsprachigen Staatsrechtslehre geäußert. Skeptisch bis ablehnend wurde eine solche Vereinbarkeit unter anderem von Hans Kelsen ${ }^{14}$ und Heinrich Triepel ${ }^{15}$ beurteilt, befürwortend beispielsweise von Gerhard Anschütz, ${ }^{16} \mathrm{Ge}$ org Jellinek ${ }^{17}$ und Carl Schmitt. ${ }^{18}$ Die Vereinbarkeit einer Wahlpflicht mit dem Grundsatz der Demokratie entsprach dabei lange Zeit der ganz überwiegenden Ansicht ${ }^{19}$ was nicht zuletzt daran erkannt werden kann, dass es in früherer Zeit nicht allein das Problem dieser Vereinbarkeit war, das diskutiert worden ist, sondern im selben oder im größeren Umfang die Frage, ob sich mit dem demokratischen Wahlrecht nicht ohnehin eine Wahlpflicht verbindet. ${ }^{20}$ Der Anschauungswandel zeigt sich sehr deutlich bei einem Blick in die staatsrechtliche Kommentarliteratur: die Mehrheit der Grundgesetzkommentare lehnt nunmehr die Vereinbarkeit einer Wahlpflicht mit dem Grundsatz der

13 Siehe dazu statt vieler Ernst-Wolfgang Böckenförde, in: Isensee/Kirchhof (Hrsg.), Handbuch des Staatsrechts, Bd. II, 3. Auflage, § 24 Rn. 35 ff.; Walter Frenz, Rechtstheorie 24 (1993), 513; Wolfram Höfling, Der Staat 33 (1994), 493 und Josef Isensee, Der Staat 20 (1981), 161; aus der älteren Literatur bleibt hierzu wertvoll Rudolf Smend, Bürger und Bourgeois im deutschen Staatsrecht, in: Staatsrechtliche Abhandlungen, 3. Auflage, S. 309.

14 Nachweise zur Position Kelsens bietet Walter Berka, VVDStRL 55 (1996), 48, 52 (dort in Fn. 11).

15 Wahlrecht und Wahlpflicht, S. 11.

16 Die Verfassung des Deutschen Reichs (Nachdruck 1965), S. 578.

17 System der subjektiven öffentlichen Rechte, S. 156.

18 Verfassungslehre, 9. Auflage, S. 254.

19 Von einer ,wohl herrschende[n] Meinung in der Literatur“ sprachen noch in den neunziger Jahren Wilfried Berg/Robert Dragunski (JuS 1995, 238, 241 m.w.N.), die selbst ebenfalls von der Verfassungsmäßigkeit einer Wahlpflicht ausgehen. In der älteren Literatur äußerten sich zugunsten der Vereinbarkeit von Wahlpflicht und Wahlfreiheit neben den bereits Genannten Heinrich Geffcken, ZfP 2 (1909), 159; Ludwig Herrfurth, DJZ 1 (1896), 23 und Georg Meyer, Das parlamentarische Wahlrecht, S. $653 \mathrm{ff}$.

20 Siehe dazu unten: II.5. 
Wahlfreiheit ab. ${ }^{21}$ Geführt wird dieselbe Diskussion inzwischen auch auf der Ebene des europäischen Rechts. In seinem Lehrbuch zur Europäischen Menschenrechtskonvention bezeichnet Christoph Grabenwarter ${ }^{22}$ die Einführung einer Wahlpflicht als Eingriff in das Recht auf freie Wahlen aus Art. 3 des 1. Zusatzprotokolls zur EMRK, der eines besonderen Rechtfertigungsgrundes bedürfe. Im Hinblick auf die Wahlen zum Europaparlament wird behauptet, dass eine Wahlpflicht bei Europawahlen nicht mit Art. 39 der EU-Grundrechtecharta zu vereinbaren sei, da diese den Grundsatz der Wahlfreiheit verletze. ${ }^{23}$ Wenn man die Konsequenzen dieser neueren Lehren zu Ende bedenkt, bedeutet dies letztlich, dass in jenen Staaten, welche die Wahlpflicht kennen, Verstöße gegen europäisches Recht über Jahrzehnte hinweg praktiziert worden sind und zum Teil, wie in Luxemburg oder Belgien, bis heute praktiziert werden. War also die früher herrschende Annahme der Vereinbarkeit einer Wahlpflicht mit dem Grundsatz der freien Wahl nur eine Art böser Traum, aus dem man inzwischen erwacht ist?

Die Frage drängt sich unweigerlich auf, wie es in den Debatten über die Wahlpflicht zu einem solchen Wechsel des vorherrschenden Standpunktes kam: was ist passiert, welche Aspekte sind neu zur Diskussion gestellt worden? Bemerkenswert ist es zunächst, dass bürgerliche Freiheitsrechte und staatsbürgerliche Mitwirkungsrechte heutzutage weniger gründlich gegeneinander abgegrenzt werden, als dies in der Dogmatik früherer Epochen getan worden ist. ${ }^{24}$ Der Grund hierfür ist leicht zu erkennen: zu einer

21 So u.a. (bezogen auf die Einführung einer Wahlpflicht ohne Änderung des Verfassungsgesetzes) Hans H. Klein, in: Herzog/Scholz/Herdegen/Klein (Hrsg.), Grundgesetz, Rn. 108 zu Art. 38; Siegfried Magiera, in: Sachs (Hrsg.), Grundgesetz, 5. Auflage, Rn. 85 zu Art. 38; Martin Morlok, in: Dreier (Hrsg.), Grundgesetz, 2. Auflage, Bd. II, Rn. 83 zu Art. 38; Bodo Pieroth, in: Jarass/Pieroth, Grundgesetz, 10. Auflage, Rn. 9 zu Art. 38 und Wolfgang Schreiber, in: Friauf/Höfling (Hrsg.), Berliner Kommentar zum Grundgesetz, Rn. 70 zu Art. 38; ablehnend außerdem Walter Frenz, ZRP 1994, 91, 94; Rolf Gröschner, VVDStRL 55 (1996), 163; Ernst Rudolf Huber, Deutsche Verfassungsgeschichte, Bd. III, 3. Auflage, S. 865; Otto Luchterhandt, Grundpflichten als Verfassungsproblem in Deutschland, S. 498; Thorsten Ingo Schmidt, Grundpflichten, S. 219; Klaus Stern, Das Staatsrecht der Bundesrepublik Deutschland, Bd. I, 2. Auflage, S. 323 und Rolf Stober, Grundpflichten und Grundgesetz, S. 65. Von der Vereinbarkeit einer Wahlpflicht mit dem Grundgesetz gehen demgegenüber aus: Wolfram Höfling, Der Staat 33 (1994), 493, 504; Detlef Merten, in: Festschrift für Johannes Broermann, S. 301, 314; ders., VVDStRL 55 (1996), 7, 24; Hans-Peter Schneider, in: Denninger/Hoffmann-Riem/Schneider/Stein (Hrsg.), Kommentar zum Grundgesetz für die Bundesrepublik Deutschland (Alternativkommentar), 3. Auflage, Rn. 66 zu Art. 38; Uwe Volkmann, in: Friauf/ Höfling (Hrsg.), Berliner Kommentar zum Grundgesetz, Rn. 29 zu Art. 20 und Reinhold Zippelius, Allgemeine Staatslehre, 14. Auflage, S. 213.

22 Europäische Menschenrechtskonvention, 4. Auflage, § 23 Rn. 103. Die gegenteilige Ansicht vertritt Dagmar Richter, in: Grote/Marauhn (Hrsg.), EMRK/GG-Konkordanzkommentar, Kap. 25 Rn. 72.

23 Dass eine Wahlpflicht nach ,allgemeinem Verständnis“ nicht mit dem Grundsatz der freien Wahl zusammengebracht werden könne, wie Art. 39 der EU-Grundrechtecharta ihn festlegt, meint Stephan Hobe, in: Tettinger/Stern (Hrsg.), Kölner Gemeinschaftskommentar zur Europäischen Grundrechte-Charta, Rn. 21 zu Art. 39; der Sache nach ebenso Siegfried Magiera, in: Meyer (Hrsg.), Charta der Grundrechte der Europäischen Union, 2. Auflage, Rn. 27 zu Art. 39.

24 Vgl. dazu auch Otto Luchterhandt, Grundpflichten als Verfassungsproblem in Deutschland, S. 496 und Hans Heinrich Rupp, in: Isensee/Kirchhof (Hrsg.), Handbuch des Staatsrechts, 3. Auflage, Bd. II, §31 Rn. 24. 
Zeit, als sich bürgerliche Gesellschaft und monarchische Obrigkeit im Kampf um die Gestaltung der politischen Ordnung befanden ${ }^{25}$ und die rechtsstaatlich-liberale Verbürgung der Freiheit mit einer demokratischen Selbstregierung des Volkes in der Praxis des staatlichen Lebens noch nicht verbunden erschien, war es offenkundig bedeutsam, Freiheitsgrundrechte und politische Mitwirkungsrechte auseinanderzuhalten. ${ }^{26}$ Dass sich diese Unterscheidung heutzutage verflüchtigt, rührt aus dem Zusammentreffen der politischen Selbstbestimmung des Volkes mit der Gewährleistung eines umfassenden Grundrechtsschutzes im freiheitlich-demokratischen Staat her.

Neu ist in der Diskussion über die Vereinbarkeit einer Wahlpflicht mit dem Grundsatz der freien Wahl indes noch ein anderer Faktor, der vielfach zum Umdenken beitragen mag, obgleich er nicht allen bewusst ist: zu verzeichnen ist auch in dieser Debatte ein verändertes (und ein weiterhin sich veränderndes) Verständnis von „Demokratie“. Im Kontext der Diskussionen über die Bedeutung der Demokratie in Zeiten der Globalisierung wird seit einem anderthalben Jahrzehnt über sogenannte postparlamentarische Formen der Demokratie nachgedacht ${ }^{27}$ - womit zwangsläufig ein verändertes und relativiertes Verständnis des Wahlrechts verbunden erscheint. Wenn erklärt wird, dass Demokratie auch anders als durch Parlamentswahlen hergestellt werden kann (ja dass Demokratie anders als durch Parlamentwahlen hergestellt werden muss), so verkommt auch das Wahlrecht schrittweise zur Marginalie. Eine Staatsrechtswissenschaft, die bis dahin gelangt ist, braucht sich um die Rechtsnatur des Wahlrechts keine hochkomplizierten Gedanken zu machen; sie kann sich damit begnügen, das Recht des Bürgers zu wählen als eines von vielen Rechten zu deuten. Vor diesem Hintergrund ist es naheliegend und leicht, jene frühere Theorie, die eine Wahlpflicht im demokratischen Staat für zulässig hält, zum Bestandteil eines veralteten und konservativen Demokratiebildes ${ }^{28}$ zu deklassieren. ${ }^{29}$

25 Dazu Rudolf Smend, Bürger und Bourgeois im deutschen Staatsrecht, in: Staatsrechtliche Abhandlungen, 3. Auflage, S. 309, 313 f.; vgl. auch Stefan Haack, JÖR 57 (2009), 301, 314 ff.

26 Vgl. dazu auch Walter Frenz, Rechtstheorie 24 (1993), 513, 524 f.; Carl Schmitt, Die geistesgeschichtliche Lage des heutigen Parlamentarismus, 8. Auflage, S. 23 und Rudolf Smend, Bürger und Bourgeois im deutschen Staatsrecht, in: Staatsrechtliche Abhandlungen, 3. Auflage, S. $309 \mathrm{ff}$.

27 Vgl. dazu die Nachweise bei Stefan Haack, Verlust der Staatlichkeit, S. 292 (dort in Fn. 28).

28 Politisch gefordert wurde die Einführung einer Wahlpflicht, je nach den Umständen des konkreten Staates und der jeweiligen Zeit, von den verschiedensten Kräften; eine Zuordnung dieses Gedankens zur einen oder anderen Seite des politischen Spektrums war und ist keineswegs möglich. So wurde die Wahlpflicht in Österreich insbesondere von Vertretern des konservativen politischen Lagers verlangt (siehe dazu Manfred Nowak, Politische Grundrechte, S. 392), während es in vielen anderen Staaten die sozialdemokratischen und sozialistischen Kräfte sind, die von einer Wahlpflicht profitieren und diese fordern (siehe dazu Malcolm Mackarras/Ian Mc Allister, Electoral Studies 18 [1999], 217, 227 ff.). Vgl. auch Alexander Vutkovich, Wahlpflicht, S. 49 f. zu den Unterschieden zwischen der Schweiz und Österreich im 19. Jahrhundert sowie Maria Gratschew, in: International Institute for Democracy and Electoral Assistance (Hrsg.), Voter Turnout in Western Europe, S. 25, 28 zu den politischen Motiven der Einführung einer Wahlpflicht in Belgien.

29 In diese Richtung Otto Luchterhandt, Grundpflichten als Verfassungsproblem in Deutschland, S. $495 \mathrm{ff}$. 


\section{Zur Vereinbarkeit einer Wahlpflicht mit dem Grundsatz der freien Wahl}

\section{Wahlfreiheit als Entscheidungsfreiheit des Wählers: zum verfassungstheoretischen Ausgangspunkt der Überlegung}

Den geläufigen Definitionen des verfassungsrechtlichen Schrifttums zufolge bedeutet der Grundsatz der freien Wahl, dass jeder mittelbare oder unmittelbare Druck auf die Entscheidungsfreiheit des Wählers ausgeschlossen sein muss ${ }^{30}$ Im Kontext der Wahlpflicht führt dies zu der Frage, was mit der „Entscheidungsfreiheit des Wählers“ gemeint ist: bezieht diese sich nur auf den Inhalt der Stimmabgabe oder umfasst sie auch die Entscheidung darüber, ob man an einer Wahl teilnimmt? ${ }^{31}$ Wo sich der Verfassungstext hierzu nicht explizit äußert (und somit auch: unter der grundgesetzlichen Ordnung), ${ }^{32}$ ergibt sich die Antwort hierauf letztlich nur aus dem Wesen des Wahlrechts und seiner verfassungsrechtlichen Bedeutung in der Demokratie. ${ }^{33}$ Um den Ausgangspunkt der Überlegung zunächst noch allgemein und voraussetzungslos zu formulieren, ließe sich sagen: ihrem Wesen zufolge unterscheidet sich die demokratische Legitimation staatlicher Hoheitsgewalt von anderen Mustern der Herrschaftsermächtigung durch die Besonderheit, dass das Volk durch eine Wahl Volksvertreter bestimmt, die es mit der Führung seines Staates beauftragt. Im folgenden wollen wir zeigen, ${ }^{34}$ weshalb das Volk in seiner Qualität als politisch entscheidende Wählerschaft selbst als ein Verfassungsorgan aufgefasst werden muss. ${ }^{35}$ Hieraus ist die Lösung des Wahlpflichtproblems zu entwickeln. Eine solche Definition des Ausdrucks „Verfassungsorgan“ im Sinne des Staatsrechts ließe sich wie folgt formulieren: ${ }^{36}$ Verfassungsorgan ist derjenige, dessen verbindliche Entscheidung bei der Verwirklichung der verfassungsrechtlichen Ordnung die

30 Vgl. statt vieler Christoph Degenhart, Staatsrecht I. Staatsorganisationsrecht, 25. Auflage, Rn. 49.

31 Vgl. dazu Wolfgang Schreiber, in: Friauf/Höfling (Hrsg.), Berliner Kommentar zum Grundgesetz, Rn. 70 zu Art. 38 und Rolf Stober, Grundpflichten und Grundgesetz, S. 64 f.

32 Otto Depenheuer (VVDStRL 55 [1996], 90, 117) sieht in dem Umstand, dass im Grundgesetz keine Wahlpflicht festgelegt ist, einen Ausdruck der begrenzten Verantwortung des Bürgers für die Gemeinschaft, die unser Verfassungsrecht präge.

33 Heinrich Triepel, ZfP 4 (1911), 597, 599.

34 Angeregt und beeinflusst wurden die folgenden Ausführungen zum Wahlrecht als einer Organkompetenz u.a. durch Heinrich Geffcken, ZfP 2 (1909), 159, 177 ff.; Georg Jellinek, System der subjektiven öffentlichen Rechte, S. 151 ff.; Carl Schmitt, Verfassungslehre, 9. Auflage, S. 254; Martin Usteri, ZSR 78 N.F. (1959), 359 a, 382 a ff. und Alexander Vutkovich, Wahlpflicht, S. 66 ff.; vgl. ferner Otto Depenheuer, VVDStRL 55 (1996), 90, 115 sowie Felix Ermacora, Allgemeine Staatslehre, Erster Teilband, S. 465. Zur Kritik an dieser Lehre siehe Otto Luchterhandt, Grundpflichten als Verfassungsproblem in Deutschland, S. 495 ff.; Thorsten Ingo Schmidt, Grundpflichten, S. 218; Zur ideengeschichtlichen Entwicklung dieses Konzepts siehe Ernst Rudolf Huber, Deutsche Verfassungsgeschichte, Bd. III, 3. Auflage, S. $864 \mathrm{f}$.

35 So ausdrücklich Wolfram Höfling, Der Staat 33 (1994), 493, 498; vgl. auch Walter Schmitt Glaeser, in: Isensee/Kirchhof (Hrsg.), Handbuch des Staatsrechts, 3. Auflage, Bd. III, § 38 Rn. 31. Vom Volk als „Verfassungs- oder Kreationsorgan“ spricht im Hinblick auf Art. 20 Abs. 2 Satz 2 GG auch BVerfGE 20, 56, 98; vom Volk als „Staatsorgan“ ist in BVerfGE 8, 104, 113 die Rede.

36 Die staatstheoretischen Hintergründe dieser Definition haben wir andernorts dargelegt; vgl. Stefan Haack, Primitive Staatstheorie, demnächst in: Der Staat. 
Verfassung selbst unmittelbar vorsieht. ${ }^{37}$ Genau dies jedoch gilt für die Wählerschaft im Hinblick auf einen Wahlakt. Als das Wesenskennzeichen der demokratischen Staatsform verstehen wir es insofern, dass das Wahlvolk und mit ihm der einzelne Wähler zum Baustein der institutionellen Architektur in der verfassungsstaatlichen Ordnung gemacht wird. ${ }^{38}$

\section{Das Wahlvolk als das Ur-Organ des demokratischen Staates}

Der eigentliche Grund dafür, das Wahlvolk in dieser Weise als ein Verfassungsorgan zu qualifizieren, liegt indes in der Frage, als was jene Entscheidung aufgefasst werden muss, die das Wahlvolk mit dem Wahlakt hervorbringt. ${ }^{39}$ Deren Charakter verdeutlicht uns folgende allgemeine Erwägung: was auf verfassungsrechtlicher Ebene oder darunter als ein Organ in der staatlichen Ordnung agiert, kann in personeller Hinsicht nur durch ein anderes Organ legitimiert sein - die Verwaltungsbehörde durch die Regierung, die Regierung durch das Parlament usw. Organe erhalten ihre personelle Legitimation nur durch Organe - ja es ließe sich sogar der Begriff des Organs in dieser Weise konkretisieren: wer über die personelle Besetzung staatlicher Organe entscheidet, muss selbst

37 Gegen entsprechende Definitionen der Organeigenschaft hatte Heinrich Triepel (ZfP 4 [1911], 597, 602) eingewandt, dass hierdurch der Begriff des Organs konturenlos würde. Er selbst definierte als „Staatsorgan, wer namens des Staates handelt, oder anders ausgedrückt: in wem wir uns den Staat selber als handelnd vorstellen müssen" (a.a.O.). Um zu einer solchen (wir geben zu: anscheinend griffigen) Definition zu gelangen, ist es allerdings nötig, vor der Unsicherheit des zugrunde gelegten Staatsbegriffs den Blick zu verschließen. Wie ist zu bestimmen, ob das „Wahlvolk“ beim Wählen „im Namen des Staates“ handelt? Wenn Triepel und die ihm folgenden Autoren mit dem Wort „Staat" die Summe der Staatsgewalt meinen, die von Amtsträgern ausgeübt wird, ist nichts gewonnen: ob das Volk als Wählerschaft dazu zählt, ist ja gerade die zu klärende Frage. Die Erörterung muss deshalb ein anspruchsvolleres Verständnis von Staatlichkeit zum Ausgangspunkt nehmen; damit jedoch erweist sich Heinrich Triepels Definitionsversuch als unpraktikabel: wenn man den Schwierigkeiten des Staatsbegriffes nicht ausweichen will, wird die Frage, wer ,im Namen des Staates“ handelt, zu einem der kniffligsten Rätsel des Staatsrechts, das sich als Definitionsgrundlage nicht eignet. Wie in vielen anderen Kontexten auch, zeigt sich hier einmal mehr das Fehlen einer juristischen Staatstheorie; vgl. Stefan Haack, Der Staat 49 (2010), 107, 109 ff. Eine solche Staatstheorie haben wir an anderer Stelle darzulegen versucht (Stefan Haack, Primitive Staatstheorie, demnächst in: Der Staat).

38 Deutlicher als anderen ist dieser Grundzusammenhang der Schweizer Staatsrechtslehre bewusst; siehe statt vieler Martin Usteri, ZSR 78 N.F. (1959), 359 a, 382 a ff. Vgl. dazu die Darstellungen und die Nachweise bei Wolfram Höfling, Der Staat 33 (1994), 493, 504 und Manfred Nowak, Politische Grundrechte, S. 154 f. Gegen die Übertragung dieser Lehren in die deutsche Dogmatik wendet sich Otto Luchterhandt, Grundpflichten als Verfassungsproblem in Deutschland, S. 497.

39 Die folgenden Ausführungen belegen, dass ein solches organschaftliches Verständnis des Wahlrechts, wie Jellinek es konzipiert hat und wie es hier in das juristische Bewusstsein zurückgeholt werden soll, alles andere als „Begriffsjurisprudenz“ ist (so jedoch Heinrich Triepel, ZfP 4 [1911], 597, 604). Es handelt sich, im Gegenteil, um ein verfassungsjuristisches Denken, das (unter verschiedenen Vorbehalten) als ein „konkretes Ordnungsdenken“ aufgefasst werden könnte (vgl. dazu auch Stefan Haack, Der Staat 49 [2010], 107, 121 ff.). 
ebenfalls ein Organ sein. ${ }^{40}$ Mit anderen Worten: es gibt innerhalb jeder neuzeitlichen staatlichen Ordnung eine Ableitungskette personeller Legitimation, die zugleich eine Kette der Übertragung von öffentlich-rechtlicher Kompetenz ist. ${ }^{41}$ Konkrete Personen, welche einem Organ angehören, werden durch ein anderes Organ - und gerade nicht durch Privatpersonen - ausgewählt und bestellt. Bis zu diesem Gedanken hat das Problem bereits vor mehr als einhundert Jahren Georg Jellinek aufgerollt. ${ }^{42}$ Gerade diese These: dass jedes Organ nur durch ein anderes Organ und nicht durch Private besetzt werden könne, wurde indes kritisiert; und Jellinek selbst hat diesen Gedankengang nicht bis zu Ende verfolgt. Zuzugeben ist der Kritik, dass eine solche Ableitungskette nicht in die Unendlichkeit fortgesetzt werden kann. Am Anfang der Organbestellungskette muss etwas anderes stehen als ein Organ.

Im neuzeitlichen Staatswesen ist dies jedoch kein Privater, ${ }^{43}$ da ein solcher Privater in einer Ableitungskette von öffentlich-rechtlichen Kompetenzen definitionsgemäß keinen Platz hat: es macht den Kern dessen aus, was man als „das Private“ versteht, dass es mit der Staatsfunktion nichts zu tun hat; und es macht den Kern dessen aus, was man als „das Öffentliche“ versteht, dass es hierbei um die Angelegenheiten und um die Ordnung des Gemeinwesens geht. Wenn der Begriff des „öffentlichen“ Rechts einen eigenen Sinn haben soll, muss man ihn von diesem Unterschied her konzipieren - mit der Konsequenz, dass auch die hier zu diskutierende Besetzung von Verfassungsorganen als eine eminent öffentlich-rechtliche Funktion erkannt werden muss, weshalb auch all diejenigen, die an einer solchen Übertragungskette öffentlich-rechtlicher Hoheitsgewalt partizipieren, ihrem Wesen nach nur Staatsorgane sein können und vom Juristen so definiert werden müssen.

Was ist es demzufolge, was sich am Anfang der Organbesetzungskette befindet, wenn es nicht der Privatbürger ist? Es ist eine Norm, welche die konkreten Personen des ersten Organs in dieser Ableitungskette erwählt und bestellt. ${ }^{44}$ Während dieser Rechtssatz in einer absoluten Monarchie sinngemäß lautet, dass dieses oder jenes Fürstenhaus die Herrscherfamilie sein soll, besagt er in der Demokratie: „Alle Staatsgewalt geht vom Volke aus. Sie wird vom Volke in Wahlen und Abstimmungen ausgeübt." Für unseren Kontext ist dabei zweierlei von Bedeutung: zum einen, dass jene Norm, indem sie eine Art von personeller Ur-Auswahl vornimmt, die Kompetenz zur Besetzung der nächsten Organe einem oder mehreren konkret existierenden Menschen zuweist ${ }^{45}$ - und zum anderen, dass diese ursprüngliche Zuweisung der Kompetenz zur Besetzung der nächsten Organe im demokratischen Staat an die Wählerschaft adressiert ist. ${ }^{46}$ Ihrer Natur nach ist dies zwangsläufig eine öffentlich-rechtliche, und zwar: eine verfassungsrecht-

40 In diesem Sinne: Georg Jellinek, System der subjektiven öffentlichen Rechte, S. 151; a.A. Heinrich Triepel, Wahlrecht und Wahlpflicht, S. 10.

41 Vgl. dazu insbesondere Martin Usteri, ZSR 78 N.F. (1959), 359 a, 390 a.

42 Vgl. Georg Jellinek, System der subjektiven öffentlichen Rechte, S. 151 ff. Dazu auch Manfred Nowak, Politische Grundrechte, S. 154.

43 So jedoch Heinrich Triepel, Wahlrecht und Wahlpflicht, S. 10.

44 Vgl. Martin Usteri, ZSR 78 N.F. (1959), 359 a, 390 a.

45 Vgl. Martin Usteri, ZSR 78 N.F. (1959), 359 a, 390 a.

46 In eine ähnliche Richtung ging Martin Usteri, ZSR 78 N.F. (1959), 359 a, 384 a f., dessen Einordnung der Organbestellungskompetenz als Befugnis zur „Normsetzung“ indes gekünstelt erscheint. 
liche Norm (ob der Verfassungstext sie fixiert, spielt dabei keine Rolle), da sie (und nur sie) unmittelbar auf der Ebene des Verfassungsrechts eine Kette öffentlich-rechtlicher Organbestellung in Gang setzt. Wenn man diesen Zusammenhang in seiner Bedeutung erkannt hat, lässt sich der Hinzutritt des Privaten kategorisch bestreiten: wie sollte man ihn im Hinblick auf das öffentlich-rechtliche Wesen einer solchen ursprünglichen Organbesetzungskompetenz überhaupt denken ${ }^{47}$

\section{Wahlvolk und Wahlakt: zur Unterscheidung von Inhaberschaft und Ausübung der Befugnis zu wählen}

Eingewandt wurde gegen diese Argumentation, dass es keineswegs zwingend sei, das Wahlvolk in seiner Gesamtheit als Verfassungsorgan zu betrachten. Möglich sei es ebensogut, das Verfassungsorgan Wahlvolk nur in jener Summe von Bürgern zu sehen, die tatsächlich abgestimmt haben. ${ }^{48}$ Erst unmittelbar mit dem Wahlakt entstünde nach dieser Lehre ein Verfassungsorgan - es wäre, genauer gesprochen, überhaupt nur im Zeitpunkt der Wahl existent. Hiergegen ist indes zweierlei zu bemerken.

Aus einer rechtstheoretischen Sicht (und dies ist unsere erste Entgegnung) ist bei der Wahrnehmung von Kompetenzen stets zwischen Inhaberschaft und Ausübung zu unterscheiden. Kompetenzen, die nur im Augenblick ihres Ausgeübtwerdens bestehen, oder, um auch hier so genau wie möglich zu sein: Kompetenzen, die erst dadurch zu solchen werden, dass man sie ausübt, sind dem Recht fremd. Rechtskompetenzen können nicht ihren eigenen Träger kreieren, sondern setzen diesen voraus. Es ist deshalb falsch anzunehmen, dass es der einzelne sei, der sich durch das Abgeben einer gültigen Stimme im Zeitpunkt des Wahlakts zum Inhaber der Organbestellungskompetenz macht - sondern es verhält sich andersherum: jene verfassungsrechtliche Norm, welche das Volk zum Wahlvolk ernennt (und damit: zum Inhaber der Kompetenz und zum Verfassungsorgan), verschafft dem einzelnen eine Berechtigung, an der Wahl teilzunehmen, und weist ihm somit die Kompetenz zu. Zwischen der verfassungsrechtlichen Norm, die bestimmt, welche konkreten Personen dem ersten Verfassungsorgan angehören, und dem hierdurch bestellten Organ kann es auch in dieser Hinsicht keinen Zwischenschritt geben.

Die zweite Entgegnung betrifft die Aussagen des Verfassungsgesetzes, das uns, die Verfassungsjuristen, anleiten muss ${ }^{49}$ wenn es darum geht, den Inhalt des Demokratieprinzips zu ermitteln. Es ist in diesem Fall der bekannte Wortlaut des Grundgesetztextes, welcher der hier verworfenen Sicht (wenn man sie auf die heutige Ordnung ummünzen will) widerspricht: Art. 20 Abs. 2 Satz 1 GG legt die Staatsgewalt in die Hände des „Volkes" - und eben nicht in die Hände der an einer konkreten Wahl beteiligten Menschen. Weil er im Bewusstsein bestimmter verfassungstheoretischer Grundzusammenhänge formuliert worden ist, lautet Art. 20 Abs. 2 Satz 1 gerade nicht (und dürfte er korrekterweise nicht lauten): „Alle Staatsgewalt geht vom Wähler aus.“ Aus diesen

47 Vgl. dazu auch Carl Schmitt, Die geistesgeschichtliche Lage des heutigen Parlamentarismus, 8. Auflage, S. 22.

48 So Heinrich Triepel, Wahlrecht und Wahlpflicht, S. 10; ders., ZfP 4 (1911), 597, 601.

49 Zum methodischen Kontext dieser Bemerkung vgl. Stefan Haack, Der Staat 49 (2010), 107, $121 \mathrm{ff}$. 
Gründen beharren wir auf dem Gesagten: mit der Zuweisung einer ursprünglichen Organbestellungskompetenz an das Volk (und an niemanden sonst) kommt im demokratischen Staat die Kette der Organbestellung in Gang ${ }^{50}$ - das Volk wählt Menschen ins Parlament, das Parlament wählt Menschen in die Regierung, die Regierung bestimmt die leitenden Beamten usw. Dass eine solche erste Personalauswahl stattfinden muss, liegt im Wesen der Dinge: jede Art von Gemeinschaft, die unter einer bestimmten verfassungsrechtlichen Ordnung besteht, hat die Frage zu lösen, wer (sprich: welche konkrete Gruppe von Menschen) am Anfang der personell-legitimatorischen Ableitungskette positioniert ist; und es löst diese Frage, die unmittelbar mit dem Akt der Verfassunggebung verknüpft ist, durch eine Norm. Genau dies bestätigt nun die eingangs gewagte Behauptung: ${ }^{51}$ der Schlüssel zur Frage der Wahlpflicht findet sich in dem Problem, wie die Idee einer bestimmten verfassungsrechtlichen Ordnung an die konkret existierende Menschengemeinschaft angeknüpft werden kann. ${ }^{52}$ Als Demokratie definieren wir dabei jene politische Ordnung, in der dieser erste konkrete Träger der weiteren Organbestellungskompetenz das Volk selbst ist. Staatsrechtlich betrachtet, ist daher nicht der Wille des Volkes, sich frei zu entfalten, das Agens der Demokratie, ${ }^{53}$ Agens der Demokratie ist der Wille des Volkes, Ur-Organ ${ }^{54}$ im Staatsganzen zu sein.

\section{Wahlfreiheit und Freiheitsgrundrechte}

Wir halten an diesem Punkt inne und können erkennen, wie weit entfernt eine solche öffentlich-rechtliche Zuständigkeit des Wahlvolks als Verfassungsorgan vom Wesen der Freiheitsgrundrechte liegt. ${ }^{55}$ Handlungsfreiheit, Berufsfreiheit oder Religionsfreiheit stecken einen Entfaltungsraum ab, in den die hoheitliche Gewalt nur insoweit ein-

50 „Die Staatsgewalt geht vom Volke aus. / - Aber wo geht sie hin? / - Ja, wo geht sie wohl hin/ - Irgendwo geht sie doch hin!“ schrieb Bertolt Brecht in seinem Gedicht „Drei Paragraphen der Weimarer Verfassung“. Wir haben soeben gesehen: sie geht zum ersten vom Volk bestellten Organ, welches in der grundgesetzlichen Ordnung der Bundestag ist. Der Verfassungstext tritt an diesem Punkt geistreicher auf als derjenige, der diese Frage gestellt hat. Überhaupt erscheint Art. 20 Abs. 2 GG in unserem Kontext aussagekräftiger, als man oft annimmt: wenn dort die ,besondere[n] Organe der Gesetzgebung, der vollziehenden Gewalt und der Rechtsprechung" erwähnt sind, lässt dies nur den Umkehrschluss zu, dass es auch ein „allgemeines“ Organ geben muss und dass dieses ,allgemeine“ Organ kein anderes als das Volk ist (vgl. dazu auch Wolfram Höfling, Der Staat 33 [1994], 493, 498). Welche andere Funktion sollte das Adjektiv „besondere“" an dieser Stelle besitzen?

51 Siehe oben: I.

52 Dazu auch Heinrich Geffcken, ZfP 2 (1909), 159, 180.

53 Vgl. jedoch Ernst-Wolfgang Böckenförde, in: Isensee/Kirchhof(Hrsg.), Handbuch des Staatsrechts, 3. Auflage, Bd. II, § 24 Rn. 37. Siehe ferner auch Martin Usteri, ZSR 78 N.F. (1959), 359 a, 376 a.

54 Von einem „Ur-Organ“ sprach in diesem Kontext auch Heinrich Geffcken, ZfP 2 (1909), 159, 178.

55 Vgl. dazu auch Wolfram Höfling, Der Staat 33 (1994), 493, 495 ff. und Heather Lardy, Oxford Journal of Legal Studies 24 (2004), 303, $311 \mathrm{ff}$. 
greifen darf, wie bestimmte öffentliche Belange dies fordern. ${ }^{56}$ Dies gilt auch dort, wo einzelne Freiheitsgrundrechte politische Dimensionen umfassen: trotz ihrer zentralen Bedeutung für Bestehen und Gedeihen der demokratischen Ordnung ${ }^{57}$ fungieren Meinungsäußerungsfreiheit, Versammlungsfreiheit oder Vereinigungsfreiheit nicht als Kompetenzen, sondern bieten grundrechtlichen Schutz. ${ }^{58}$ Wo der einzelne ein entsprechendes Freiheitsrecht ausübt, tritt nicht das Volk als Verfassungsorgan auf (es tritt dort überhaupt kein Organ auf), sondern es handeln individuelle Akteure, Interessengruppen, Parteien - mit einem Wort: es agieren die einzelnen als die „Gesellschaft" ${ }^{59}$ Durch die unzutreffende These, dass es sich auch beim Wahlrecht nicht um eine Kompetenz, sondern um eine Art Grundrecht handelt, ${ }^{60}$ würde die verfassungsrechtliche Rolle des Bürgers als Wähler nicht aufgewertet, sondern heruntergestuft. Sie fiele aus der Sphäre des Staatsorganisationsrechts heraus. Das Volk als das Ur-Organ des demokratischen Staates würde zum verfassungsrechtlichen nullum. ${ }^{61}$

\section{Drei Missverständnisse: zur Unterscheidung von objektivem Recht und subjektivem Recht, zur Rechtsposition des einzelnen Wählers und zum Pflichtcharakter von Kompetenzen}

Die hier vorgetragene Ansicht, dass das Recht zu wählen seinem Wesen nach eine Organkompetenz von Verfassungsrang bildet, ist an dieser Stelle gegen drei Missverständnisse zu schützen, wie sie die Kritik an einer solchen organschaftlichen Begründung des Wahlrechts nicht selten verursacht. Nicht gleichzusetzen ist die von uns betonte Antinomie von Grundrecht und Organkompetenz mit der Unterscheidung von objekti-

56 Anschaulich hierzu Rudolf Smend, Bürger und Bourgeois im deutschen Staatsrecht, in: Staatsrechtliche Abhandlungen, 3. Auflage, S. 309, 313 f. (der dabei das liberale Grundrechtsverständnis in Deutschland von der französischen Menschenrechtsdoktrin unterscheidet).

57 Näher hierzu Christoph Degenhart, in: Dolzer/Kahl/Waldhoff/Graßhof(Hrsg.), Bonner Kommentar zum Grundgesetz, Rn. 1 ff. zu Art. 5 Abs. 1 und 2 und Walter Schmitt Glaeser, in: Isensee/Kirchhof (Hrsg.), Handbuch des Staatsrechts, 3. Auflage, Bd. III, § 38 Rn. 11 ff.

58 Siehe dazu Ernst-Wolfgang Böckenförde, in: Isensee/Kirchhof (Hrsg.), Handbuch des Staatsrechts, 3. Auflage, Bd. II, § 24 Rn. 37; ferner auch Alexander Vutkovich, Wahlpflicht, S. 74.

59 Vgl. dazu auch Josef Isensee, Der Staat 20 (1981), 161, $165 \mathrm{ff}$.

60 So z.B. Walter Frenz, ZRP 1994, 91, 93 und Rolf Stober, Grundpflichten und Grundgesetz, S. 65 (,grundrechtsähnliches Recht“", das zu behandeln sei wie ein Freiheitsgrundrecht).

61 Die ausführlich begründete Ansicht von Walter Frenz (Rechtstheorie 24 [1993], 513), dass die Wahlen nicht zur Sphäre des Staates, sondern zur Sphäre des Gesellschaftlichen gehören, beruht weitestgehend auf einer unreflektierten Verwendung des Ausdruckes „Staat“ (vgl. dazu schon oben: Fn. 37). Obgleich auch Walter Frenz (a.a.O., S. 521) anerkennt, dass „Staat“ und „Gesellschaft“ im demokratischen Staat des Hier-und-Heute ,versöhnt“ sind (so wörtlich ders.), übersieht er, dass gerade dies es erfordert, jenen verzerrten Staatsbegriff zu korrigieren, den die liberale Staatstheorie eines 19. Jahrhunderts (als Staatsbegriff einer Übergangsepoche) geprägt hat. Mit anderen Worten: solange man mit einem Staatsbegriff operiert, der nichts anderes bedeutet als „Hoheitsgewalt“, um ihn der Freiheitssphäre des einzelnen gegenüberzustellen, lässt sich die Rolle des Bürgers im Staat, insbesondere im Hinblick auf dessen demokratische Mitwirkungsrechte, nur fragmentarisch und zerrbildartig erfassen. Vgl. dazu auch Stefan Haack, Der Staat 49 (2010), 107, 113 ff. und ders., JÖR 57 (2009), 301, 314 ff. 
vem Recht und subjektivem Recht, wie dies sehr häufig getan worden ist. ${ }^{62}$ Es ist möglich (und nicht allein im Prozessrecht verbreitet), auch im Hinblick auf Organkompetenzen von „,subjektiven Rechtspositionen“ zu sprechen ${ }^{63}$ - so dass beispielsweise im Kommunalrecht gesagt werden kann, eine Maßnahme des Bürgermeisters verletze die „subjektiven Rechte“ eines Gemeinderatsmitglieds; ${ }^{64}$ ebenso wie auf der Ebene des Verfassungsrechts dem Parlament „,subjektive Rechte“ gegenüber der Regierung zuerkannt werden müssen. ${ }^{65}$ Dies so zu nennen, hat durchaus seinen Sinn, da es sich bei „subjektiven Rechten“ dieser Art um Rechtspositionen handelt, die bestimmten Trägern im Rahmen ihrer öffentlich-rechtlichen Funktionswahrnehmung zugeteilt sind und von diesen als eigene Rechte geltend gemacht werden können. Von einer öffentlich-rechtlichen Kompetenz des Wahlvolks zu sprechen, heißt daher nicht automatisch, dass es sich hierbei nur um objektives Recht handeln könne.

Eng hiermit ist ein zweites Missverständnis verbunden, welches das Verhältnis der Rechtsposition des einzelnen Wählers zur Rechtsposition des Wahlvolks im ganzen betrifft. Hierzu wurde vereinzelt behauptet, dass das Wahlrecht nur dem Volk in seiner Gesamtheit und nicht dem einzelnen zusteht - die Rechtsposition eines Wählers wurde demzufolge nur als eine Art von Rechtsreflex interpretiert. ${ }^{66}$ Zutreffend erfassen lässt sich dieses Verhältnis, wenn man die Situation des Wahlvolks als Verfassungsorgan mit der Situation anderer Verfassungsorgane vergleicht. Dass Verfassungsorgane aus Untergliederungen bestehen, die ihrerseits über eigene, abwehrfähige Rechtspositionen verfügen, ist nichts Untypisches - man denke wiederum an das Parlament mit seinen Fraktionen und den einzelnen Abgeordneten. Das Recht zu wählen, sprich: das Recht, beim Wahlakt seine Stimme abzugeben, ist hierbei eine Befugnis, die ihrem Wesen zufolge der einzelne Wähler, und somit ein Organteil, besitzt ${ }^{67}$ - ebenso wie das Rederecht und das Recht, an den Sitzungen teilzunehmen, in erster Linie als Befugnisse des einzelnen Abgeordneten eingestuft werden müssen. Die Vorstellung, dass der einzelne Wähler sein Recht zu wählen als ein Organteil des Wahlvolks erhält, ${ }^{68}$ ist auch insofern plausibel begründbar.

62 Die Gegenüberstellung von „subjektiven Rechten“ und „Funktionen“ findet sich bei Manfred Nowak, Politische Grundrechte, S. $152 \mathrm{ff}$.

63 So bereits Heinrich Geffcken, ZfP 2 (1909), 159, 178.

64 Von „subjektiven Rechten“, die im Kommunalverfassungsstreit geltend gemacht werden können, sprechen u.a. Martin Burgi, Kommunalrecht, 2. Auflage, § 13 Rn. 13 (,subjektive Positionen des Innenrechts“); Max-Emanuel Geis, Kommunalrecht, § 26 Rn. 16 sowie Eberhard Schmidt-Aßmann/Hans Christian Röhl, in: Schmidt-Aßmann/Schoch (Hrsg.), Besonderes Verwaltungsrecht, 14. Auflage, 1. Kap. Rn. 84.

65 Von „subjektiven Rechten“, die im Organstreitverfahren geltend gemacht werden können, sprechen u.a. Christoph Degenhart, Staatsrecht I. Staatsorganisationsrecht, 25. Auflage, Rn. 752; Christian Hillgruber/Christoph Goos, Verfassungsprozessrecht, 2. Auflage, Rn. 367 und Christian Pestalozza, Verfassungsprozessrecht, 3. Auflage, § 7 Rn. 29.

66 In diese Richtung Georg Jellinek, System der subjektiven öffentlichen Rechte, S. 152. Weitere Nachweise zu jener Ansicht finden sich bei Manfred Nowak, Politische Grundrechte, S. $153 \mathrm{f}$.

67 Vom Wähler als einem „Teilorgan“ sprach Georg Jellinek, System der subjektiven öffentlichen Rechte, S. 151. Insofern trifft auch die Aussage Otto Depenheuers zu, dass der Bürger im Wahlakt „Amtsträger" sei (VVDStRL 55 [1996], 90, 115). Ähnlich Felix Ermacora, Allgemeine Staatslehre, Erster Teilband, S. 465 und Heather Lardy, Oxford Journal of Legal Studies 24 (2004), 303, 317.

68 Ähnlich Wolfram Höfling, Der Staat 33 (1994), 493, 506. 
Das dritte Missverständnis, das sich sowohl bei Befürwortern ${ }^{69}$ als auch bei Gegnern ${ }^{70}$ der hier vertretenen Auffassung nachweisen lässt, betrifft den Pflichtcharakter der Kompetenz. Das Recht zu wählen als Organkompetenz zu betrachten, heißt nicht ohne weiteres, es auch als Pflicht behandeln zu müssen, wie dies früher zum Teil gesagt worden ist. Eine öffentlich-rechtliche Kompetenz innezuhaben besagt nichts darüber, wann und wie oft man sie ausüben muss ${ }^{71}$ - auch ein sporadisches Nichtstun kann bei besonderen Gründen von ihr umfasst sein. Eine solche Besonderheit, welche im Falle des Wahlrechts einem unbedingten Pflichtcharakter der Kompetenz widerspricht, ergibt sich aus Form und Funktionen der Stimmabgabe in einer demokratischen Ordnung: es handelt sich hierbei um eine Organkompetenz, die ihrem Sinn und Zweck nach durch einen inneren und unverfälschten Entscheidungsakt ausgeübt wird, ${ }^{72}$ der grundsätzlich nicht erzwingbar sein kann, und der, wenn man ihn dennoch erzwingen wollte, keinerlei Wert mehr besäße. In diesem Kontext steht die Freiheit der Wahl. Wie dies beides: Kompetenzcharakter und Entscheidungsfreiheit, rechtlich miteinander in Einklang gebracht werden kann, ist nichts, was sich unmittelbar aus dem Wesen des demokratischen Wahlrechts ableiten lässt - diesen Einklang herzustellen, ist die Aufgabe der Wahlgesetzgebung. Der Freiheit der Wahl genügt es, dass irgendeine Möglichkeit der Stimmenthaltung besteht, und diese besteht bei einer geheimen Wahl praktisch immer. ${ }^{73}$ Das zusammengenommen bedeutet: allein aus dem Kompetenzcharakter des Wahlrechts ergibt sich noch keine Pflicht des einzelnen, an einer Wahl teilzunehmen; es ist jedoch mit dem Kompetenzcharakter des Wahlrechts vereinbar, eine solche Pflicht zu statuieren - dies sowohl durch eine Änderung der Verfassung als auch durch einfaches Gesetz. ${ }^{74}$

\section{Ein möglicher Einwand: menschenrechtliche Gehalte im Kontext der Demokratiediskussion}

Möglich bleibt gegen all das Gesagte noch ein grundsätzlicher Einwand: der Einwand nämlich, dass es eine ganze Reihe von Gründen gäbe, das Recht auf demokratische

69 So z.B. bei Carl Schmitt, Verfassungslehre, 9. Auflage, S. 254 sowie Alexander Vutkovich, Wahlpflicht, S. 74 (u.ö.); ansatzweise in diese Richtung auch Felix Ermacora, Allgemeine Staatslehre, Erster Teilband, S. 465 und Heather Lardy, Oxford Journal of Legal Studies 24 (2004), 303, 317; vgl. auch Georg Meyer, Das parlamentarische Wahlrecht, S. 654.

70 So z.B. bei Thorsten Ingo Schmidt, Grundpflichten, S. 218.

71 Ähnlich Heinrich Geffcken, ZfP 2 (1909), 159, 182; Heinrich Triepel, Wahlrecht und Wahlpflicht, S. 10; ders., ZfP 4 (1911), 597, 601. Zu weit geht es freilich, wenn Ernst Rudolf Huber (Deutsche Verfassungsgeschichte, Bd. III, 3. Auflage, S. 865 f.) gerade aus dem „öffentlichen Status" eine negative Wahlfreiheit, sprich also: die Unvereinbarkeit einer Wahlpflicht mit einer freiheitlichen Verfassung, ableiten will.

72 Vgl. Walter Frenz, Rechtstheorie 24 (1993), 513, 516 und Ernst Rudolf Huber, Deutsche Verfassungsgeschichte, Bd. III, 3. Auflage, S. 865.

$73 \mathrm{Zu}$ der faktischen Möglichkeit des Stimmenthaltens bei einer Wahl, die geheim ist, und den Bezügen zur Freiheit der Wahl vgl. Detlef Merten, in: Festschrift für Johannes Broermann, S. 301, 310; Georg Meyer, Das parlamentarische Wahlrecht, S. 657; Martin Morlok, in: Dreier (Hrsg.), Grundgesetz, 2. Auflage, Bd. II, Rn. 83 zu Art. 38 und Reinhold Zippelius, Allgemeine Staatslehre, 14. Auflage, S. 213.

74 Im Ergebnis ebenso Detlef Merten, in: Festschrift für Johannes Broermann, S. 301, 315. 
Wahlen auch als ein Menschenrecht zu verstehen ${ }^{75}$ - mit der unausweichlichen Konsequenz für unser Problem, dass es sich beim Wahlrecht auch um ein Recht des Individuums handeln müsste, welches dann in seiner negativen Dimension davor schützt, zur Wahlteilnahme gezwungen zu werden. Und in der Tat: wenn es einen solchen menschenrechtlichen Anknüpfungspunkt gibt, müsste er dann nicht die Ableitung aus der Stellung des Wahlvolks als Verfassungsorgan überdecken? Seiner Natur nach ist dieses Problem ein Problem der Rechtstheorie: es betrifft die Geltungsgründe des Rechts - und es bedarf aus diesem Grund einer rechtstheoretischen Antwort. Diese Antwort lautet, dass erst aus einer konstituierten Ordnung heraus ablesbar ist und juristisch geklärt werden kann, welche Rollen das Volk und der einzelne spielen - Befugnisse, Rechte und Zuständigkeiten, die man im Hinblick auf das politische Leben innerhalb einer Ordnung besitzt, können nur ordnungsbedingte Befugnisse sein. ${ }^{76}$ Es ist der Wille einer konkreten menschlichen Gemeinschaft, unter einer demokratischen Herrschaft zusammenzuleben, der den demokratischen Verfassungsstaat hervorbringt und perpetuiert. ${ }^{77}$ Dies jedoch heißt, dass ein Recht zu wählen aus den oben erörterten Gründen nichts anderes sein kann als jene öffentlich-rechtliche Kompetenz eines Wahlvolkes, über die Besetzung von Organen zu entscheiden, womit eine Wahlpflicht vereinbar erscheint.

\section{Zur Vereinbarkeit einer Wahlpflicht mit den Freiheitsgrundrechten}

Dass eine Wahlpflicht wegen des Kompetenzcharakters der Befugnis zu wählen dem Grundsatz der Wahlfreiheit nicht widerspricht, bedeutet freilich noch nicht, dass sie mit dem Grundgesetz insgesamt zu vereinbaren ist. Fragen ließe sich nämlich, ob eine Wahlpflicht, indem sie ein Verhalten abverlangt, möglicherweise gegen die Freiheitsgrundrechte verstößt - wie zum Beispiel gegen die Meinungsäußerungsfreiheit gemäß Art. 5 Abs. 1 Satz 1 GG, gegen die Gewissensfreiheit gemäß Art. 4 Abs. 1 GG oder gegen die allgemeine Handlungsfreiheit gemäß Art. 2 Abs. 1 GG. (Hierbei ist klar, dass dies nur dann diskutiert werden kann, wenn die Wahlpflicht durch ein einfaches Gesetz eingeführt werden soll.) Dass staatsbürgerliche Pflichten in die Grundrechte eingreifen und anhand dieser Normen kontrolliert werden müssen, ist aus vielen anderen Zusammenhängen bekannt - genannt sei hier nur die Pflicht zum Entrichten von Steuern.

Bevor man jedoch beginnt, nach der entsprechenden Lösung für den Fall der Wahlpflicht zu suchen, empfiehlt es sich innezuhalten, um die Bedingungen zu überprüfen, unter welchen die Frage nach der Vereinbarkeit einer Wahlpflicht mit den Freiheitsgrundrechten überhaupt nur gestellt werden darf. Wer nämlich meint, dass es sich beim

75 In diese Richtung z.B. Walter Frenz, Rechtstheorie 24 (1993), 513, 529 f.; ders., ZRP 1994, 91, 92 f. und Otto Luchterhandt, Grundpflichten als Verfassungsproblem in Deutschland, S. 497; zu den ideengeschichtlichen Wurzeln dieser Lehren im Naturrechtsdenken Manfred Nowak, Politische Grundrechte, S. 155 f. und Carl Schmitt, Die geistesgeschichtliche Lage des heutigen Parlamentarismus, 8. Auflage, S. 16 ff. Vgl. dazu ferner Alexander Vutkovich, Wahlpflicht, S. $68 \mathrm{f}$.

76 Als unzutreffend, zumindest jedoch als missverständlich zurückzuweisen ist daher die Annahme von Walter Frenz (Rechtstheorie 24 [1993], 513, 523), dass der demokratische Legitimationsvorgang selbst keiner Herleitung bedürfe.

77 Ausführlich hierzu Stefan Haack, Verlust der Staatlichkeit, S. 283 ff. 
Wahlrecht nicht um eine Kompetenz, sondern um ein Grundrecht oder um etwas den Grundrechten Ähnliches handelt, der kann eine Wahlpflicht nicht an den Freiheitsgrundrechten messen (oder um es aus der Perspektive eben dieser Rechtsansicht zu formulieren: der kann eine Wahlpflicht nicht an anderen Freiheitsgrundrechten als an der Wahlfreiheit messen), da Art. 38 Abs. 1 GG diesen Grundrechten gegenüber stets die lex specialis sein müsste. ${ }^{78}$ Nur wer die hier vorgetragene Auffassung teilt, kann zu einer solchen Prüfung am Maßstab der Freiheitsgrundrechte gelangen: Wahlrecht und Freiheitsgrundrechte erscheinen uns als wesensverschieden. ${ }^{79}$

Es liegt indes auf der Hand, dass auch durch die Wahlpflicht eine Meinungskundgabe ${ }^{80}$ nicht abverlangt wird: ${ }^{81}$ bei jeder geheimen Wahl ist eine Stimmenthaltung formal oder formlos - ohne weiteres möglich. ${ }^{82}$ Auch die Freiheit des Gewissens ist aus diesem Grund nicht betroffen ${ }^{83}$ - wer es nicht mit seinem Gewissen vereinbaren kann, an einer Wahl teilzunehmen, besitzt die Option, seine Wahlstimme ungültig werden zu lassen. Die Wahlpflicht greift somit letztlich nur in die allgemeine Handlungsfreiheit des einzelnen aus Art. 2 Abs. 1 GG ein. ${ }^{84}$ Dass die Anforderungen an die Rechtfertigung dieses Eingriffs erfüllbar erscheinen, ist nicht allzu schwer zu erkennen: als ein legitimes Regelungsziel kann hierbei die demokratische Legitimation der Verfassungsorgane und somit ein höchstrangiges Rechtsgut unserer freiheitlich-demokratischen Staatsordnung angeführt werden. ${ }^{85} \mathrm{Ob}$ die Wahlpflicht sich eignet, die Wahlbeteiligung zu erhöhen und dadurch die demokratische Ordnung zu stärken, ist unter Politologen umstrit-

78 Dogmatisch unklar bleibt Walter Frenz (ZRP 1994, 91, 92), wenn er Art. 38 Abs. 1 GG als eine „Positivierung des in Art. 4 Abs. 1 GG angelegten aktiven Bürgerrechts“ interpretiert.

79 Siehe dazu oben: II.4.

80 Zur Frage, ob die Stimmabgabe bei einer Wahl überhaupt eine Meinungskundgabe darstellt, siehe Walter Frenz, ZRP 1994, 91, 92 und Sean Matsler, Southern California Law Review 76 (2003), 953, 971 (für das US-amerikanische Recht).

81 Im Ergebnis ebenso Sean Matsler (Southern California Law Review 76 [2003], 953, 969 ff.) für das US-amerikanische Recht; a.A. wohl Wolfgang Schreiber, in: Friauf/Höfling (Hrsg.), Berliner Kommentar zum Grundgesetz, Rn. 70 zu Art. 38. Vgl. dazu auch Heather Lardy, Oxford Journal of Legal Studies 24 (2004), 303, $318 \mathrm{f}$.

82 Siehe dazu oben: II.5. (mit Fn. 73). Für das US-amerikanische Recht verlangt Sean Matsler, Southern California Law Review 76 (2003), 953, 974 ff., eine ausdrückliche „None-of-theabove"-Option auf dem Abstimmungszettel.

83 Ähnlich für das britische Recht Heather Lardy, Oxford Journal of Legal Studies 24 (2004), 303, 318.

84 Zur Frage, ob sich aus einer allgemeinen Verbürgung der Freiheit durch die Verfassung das Recht ableiten lässt, nicht an der Wahl teilzunehmen, siehe Heather Lardy, Oxford Journal of Legal Studies 24 (2004), 303, 307 (dort in Fn. 10), die dies für das britische Recht mit „,nein" beantwortet.

85 Näher dazu Wilfried Berg/Robert Dragunski, JuS 1995, 238, 242; vgl. auch Felix Ermacora, Allgemeine Staatslehre, Erster Teilband, S. 464 und Lisa Hill, Political Studies 50 (2002), 80, 82; dies., Journal of Sociology 36 (2000), 30, $31 \mathrm{f}$. 
ten; ${ }^{86}$ statistische Studien ${ }^{87}$ deuten jedoch mehrheitlich in diese Richtung. Zur Geeignetheit einer solchen Verpflichtung im Sinne des Verhältnismäßigkeitssatzes genügt es, dass die intendierte Wirkung zumindest als möglich erscheint - der Gesetzgeber besitzt hierbei eine Einschätzungsprärogative ${ }^{88}$ deren Grenzen in diesem Fall nicht erreicht sind. Gegen die Vereinbarkeit einer Wahlpflicht mit den Freiheitsgrundrechten bestehen insofern keine Bedenken. ${ }^{89}$

\section{Zur Wahlpflicht bei anderen Wahlen als Bundestagwahlen}

Bei allem, was bis zu diesem Punkt zur Vereinbarkeit einer Wahlpflicht mit dem Grundsatz der Demokratie und den Freiheitsgrundrechten dargelegt worden ist, sind wir, ohne dies ausdrücklich ausgesprochen zu haben, von einer ganz bestimmten Art der Wahl ausgegangen: nämlich von der Parlamentswahl im demokratischen Staat. Dies jedoch legt es nahe zu fragen, inwiefern die hier angebotenen Gründe für eine solche Vereinbarkeit auf andere Wahlen erstreckt werden können. Der Grundsatz der freien Wahl ist bekanntlich auch für diese Wahlen verfassungsrechtlich verbindlich (vgl. Art. 28 Abs. 1 Satz 2 GG). Gilt das Gesagte also in entsprechender Weise für die Wahlen zum Landtag und $\mathrm{zu}$ den Vertretungen in den Kommunen? ${ }^{90}$ Oder umgekehrt formuliert: weist möglicherweise die gesamtstaatliche Parlamentswahl ein Spezifikum auf, das für die Organqualität der Wählerschaft maßgeblich ist? Als was agiert beispielsweise die Bürgerschaft einer Gemeinde, wenn sie den Gemeinderat wählt? Aus jenen theoretischen Gründen, die wir soeben ausgeführt haben, ${ }^{91}$ meinen wir auch hier, dass die Gemeindebürger bei der entsprechenden Wahl als Organ, und zwar in diesem Fall: als das

86 Von positiven Wirkungen auf die Wahlbeteiligung gehen u.a. aus: Lisa Hill, Political Studies 50 (2002), 80, 82; Arend Lijphart, American Political Science Review 91 (1997), 1 f. sowie Malcolm Mackarras/Ian Mc Allister, Electoral Studies 18 (1999), 217, 218; vgl. auch Maria Gratschew, in: International Institute for Democracy and Electoral Assistance (Hrsg.), Voter Turnout in Western Europe, S. 25, 26 f. und Sean Matsler, Southern California Law Review 76 (2003), 953, $962 \mathrm{ff}$.

87 Siehe insbesondere Wolfgang Hirczy, Electoral Studies 13 (1994), 64 (zum Beispiel der Republik Österreich); Marc Hooghe/Koen Pelleriaux, Electoral Studies 17 (1998), 419 (zum Beispiel Belgiens - der Fokus liegt hierbei auf dem Einfluss der Wahlpflicht bei unterschiedlichen Bevölkerungsgruppen) und Galen Irwin, Comparative Political Studies 7 (1974), 292 (zum Beispiel der Niederlande). Weiteres Zahlenmaterial bietet Arend Lijphart, American Political Science Review 91 (1997), 1, 8 ff. Statistiken zur Wahlbeteiligung in Belgien vor und nach Einführung der Wahlpflicht im Jahre 1893 finden sich bei Alexander Vutkovich, Wahlpflicht, S. $58 \mathrm{ff}$.

88 So auch Wilfried Berg/Robert Dragunski, JuS 1995, 238, 242; allgemein zur Einschätzungsprärogative des Gesetzgebers im Rahmen der Geeignetheitsprüfung Christoph Degenhart, Staatsrecht I. Staatsorganisationsrecht, 25. Auflage, Rn. 401.

89 Einen unverhältnismäßigen Eingriff in Art. 2 Abs. 1 GG (oder in Art. 38 Abs. 1 GG - dies wird ausdrücklich offen gelassen) nimmt demgegenüber Otto Luchterhandt an (Grundpflichten als Verfassungsproblem in Deutschland, S. 497 f.). Argumente zur Gewichtung im Hinblick auf den Eingriff in die (Handlungs-) Freiheit des Wählers finden sich u.a. bei Lisa Hill, Political Studies 50 (2002), 80, 90 f. und Alexander Vutkovich, Wahlpflicht, S. 89 ff.

90 Zur (deutlich komplexeren) Frage einer Wahlpflicht bei Europawahlen legen wir zu gegebener Zeit eine gesonderte Abhandlung vor.

91 Siehe oben: II.2. 
Ur-Organ ihrer Gemeinde, auftreten. Die Organqualität der Bürgerschaft folgt auch hier aus dem Wesen der Wahlentscheidung, die darauf abzielt, das Personal des nächsten Organs zu bestimmen: in diesem Fall die Mitglieder der Gemeindevertretung. Entsprechendes gilt für das Wahlvolk des Landes im Hinblick auf die Wahlen zum Landtag. Auch Teilmengen des Volkes können somit als Organe ins Spiel gebracht werden: es ist durchaus vorstellbar und die Praxis des staatlichen Lebens beweist es, dass sich eine Rechtsordnung nicht damit begnügt, an die konkret vorhandene Menschengemeinschaft nur in einer einzigen Hinsicht und an einem einzelnen Punkt anzuknüpfen; ja es ist heutzutage kaum noch anders denkbar, als dass mehrere dieser Anknüpfungspunkte bestehen. Dass es solcher weiteren personell-legitimatorischen Ableitungsketten bedarf, beruht auf den zugrunde liegenden Modifikationen der demokratischen Staatsform, die unmittelbar im Verfassungsrecht angelegt sind: ${ }^{92}$ sei es in der Gestalt des kommunalen Selbstverwaltungsrechts, sei es in der Gestalt des Föderalismus oder sei es in Gestalt sonstiger verselbständigter hoheitlicher Akteure. Ebenso wie bei der Bundestagswahl ist es nicht der Private, der sich am Anfang dieser zusätzlichen Organbestellungsketten befindet; statt dessen finden wir auch an dieser Stelle öffentlich-rechtliche Normen, die konkrete Träger der Organbestellungskompetenz definieren. Alles oben Gesagte lässt sich insofern auch für diese Wahlen behaupten.

\section{Fazit}

Als Fazit der Überlegungen zur Vereinbarkeit einer Wahlpflicht mit den Verfassungsgrundsätzen halten wir vier Punkte fest: in verfassungsrechtlicher Hinsicht haben wir gesehen, dass entgegen zahlreicher Stimmen in der Literatur eine Wahlpflicht weder den Freiheitsgrundsätzen noch dem Demokratieprinzip widerspricht. Dies gilt nicht allein für die gesamtstaatliche Bundestagswahl, sondern ebenso für andere Wahlen wie die Kommunalwahl und die Wahlen zum Landtag. Die verfassungstheoretische Begründung dafür haben wir in der ursprünglichen Anknüpfung der demokratischen staatlichen Ordnung als abstrakte Idee an die konkret vorhandene Menschengemeinschaft gefunden. Diese demokratische Ordnung wiederum ist es, die dem einzelnen sein Recht zu wählen verschafft.

92 Ausführlich hierzu Stefan Haack, Verlust der Staatlichkeit, S. 308 ff.; ders., JÖR (2009), 301, 326. 